\title{
Actividades de Investigación y Docentes en las Areas del Análisis, Diseño, Construcción y Automatización de Sistemas Mecánicos en el Departamento de Ingeniería Mecánica-Uniandes.
}

\section{Roque Saltarén P.}

\section{Resumen}

E artículo presenta de manera breve las actividades académicas y de investigación, relacionadas con las siguientes áreas, básicas dentro de los planes de desarrollo del Departamento de Ingeniería Mecánica.

- Análisis y diseño asistido por computador de sistemas mecánicos: Modelaje y simulación cinemática y dinámica de mecanismos. Vibraciones yDiseño.

- Diseño, construcción y automatización de sistemas mecánicos (Robots, máquinas herramientas).

- Ingeniería de manufactura, enfoques sobre las modernas tecnologías de manufactura y estructuturación académica para la formación científica y técnica en estas áreas prioritarias para el país. resultados positivos formativos tanto de los profesoresinvestigadores como del talento de los futuros ingenieros que van a actuar y corresponder a las expectativas de la industria.

En este sentido el Departamento de Ingeniería Mecánica ha estructurado un grupo de profesores-investigadores en las áreas mencionadas, cuyo trabajo es respaldado por proyectos de investigación y desarrollo de prototipos que están fuertemente ligados a la docencia, de tal forma que le evolución de estas investigaciones está apoyada por la participación de estudiantes y el entusiasmo de tratar problemas muy actuales, como son la Robótica y el control numérico de máquinas herramientas. Evidentemente en elfondo existe un gran respaldo a la docencia sobre las nuevas tecnologías puesto que un investigador que entiende lo que está haciendo naturalmente sabe explicarlo.

\begin{tabular}{|l|l|}
\hline 20 & $\begin{array}{l}\text { Roque Saltarén } \mathrm{P} . \\
\text { Ingeniero Mecánico, Universidad del Valle, } \\
\text { Magister en Ingeniería Eléctrica, } \\
\text { Uniandes. Profesor de Ingeniería } \\
\text { Mecánica, Uniandes. Area de } \\
\text { Especialización Control y Automatización } \\
\text { Industrial. }\end{array}$ \\
\hline
\end{tabular}

Los proyectos que se describen posteriormente han dispuesto de un efectivo apoyo por parte del CIFI, y del Departamento de

\section{Introducción}

El problema de seguir el paso a la evolución de los conceptos del análisis, diseño y realización de los modernos sistemas y procesos mecánicos, es un aspecto inquietante que obliga a discernir sobre un acertado enfoque de ideas y objetivos que den lugar a
Ingeniería Mecánica en el caso del Robot Mecandes-l y de la conversión a control numérico de un torno multipropósito convencional.

En el caso del prototipo de Robot Industrial Andes-1, este es un proyecto del grupo DFAC(Dir. Dr. José Tiberio H.), proyecto PIDAI el cual se lleva a cabo con el respaldo del CIFl y COLCIENCIAS.

\section{Aspectos investigativos $y$ docentes}

Es fascinante como aunque desde los años 1700's era conocida la aplicación de los multiplicadores de Lagrange a las ecuaciones del movimiento de sistemas mecánicos con restricciones, solo hasta 1981 se reconoció completamente que esas ecuaciones no deben ser tratadas como ecuaciones diferenciales. A partir de entonces se desató una intensa actividad investigativa sobre métodos que integraban ecuaciones dinámicas diferenclales- algebrálcas, las nuevas topologías de la representación de los sistemas mecánicos y su simulación computacional aplicando métodos numéricos. En ese momento se lograron inmensos avances en esas áreas y recientemente ya existe software comercial basado en las técnicas de coordenadas generalizadas. Precisamente este nuevo enfoque se ha adoptado para los cursos de Dinámica, Mecanismos y

Vibraciones, con lo cual se están formando estudiantes que posteriormente de acuerdo a su interés se vincularán al grupo de investigación para el análisis y diseño de sistemas mecánicos asistido por computador. 
En la figura 1, se puede ver uno de los proyectos realizados por estudiantes en los cursos de Dinámlca y Mecanismos. Este proyecto permite simular el comportamiento cinemático y dinámico en el espectro del tiempo, de un mecanismo para cinematógrafo.

Se puede notar que no ha sido dificll para los estudiantes lograr la animación en tiempo real del mecanismo mencionado.

Las técnicas de simulación permiten calcular la posiclón. velocidad, aceleración, fuerzas de reacción y torques de accionamiento para todas las barras en el tiempo, parámetros fundamentales para establecer las condiciones de frontera necesarias para el análisis y diseño por elementos finitos de cada uno de los elementos mecánicos.

Otros proyectos que vale la pena menclonar son: estudio de un mecanismo para retroexcavadoras, diseño de una máquina paletizadora de gaseosas, estudio de un a máquina brochadora, estudio de dos tipos de trituradoras de pledras entre otros.

En los aspectos investigativos acoglendo las iniciativas del Decano de la Facultad y del Director del Departamento de Ingenlería de Sistemas sobre desarrollos en Software Educativo. el Departamento de Ingeniería Mecánica está participando activamente bajo la coordinación del Profesor Investigador Felipe Botero, iniciando un proyecto de investigación para el desarrollo formal de técnicas

computacionales para la simulaclón de sistemas mecánlcos. captándo el interés e iniclativa en esta área de estudlantes de la facultad.

\section{Diseño, Construcción y Automatización de Sistemas Mecánicos.}

En tanto que el área de Interés menclonada arriba sugiere la disposiclón de modernas herramlentas para el análisls $y$ decisión, son necesarias las reallzaciones fisicas de prototipos que toquen necesidades del sector industrial colombiano, cuyo feliz término dé lugar a la industrialización de las soluciones planteadas, aquí se pueden mencionar tres proyectos que se desarrollan actualmente con la participación del Departamento de Ingenbleria Mecánica.

\section{* Caso del Robot Mecandes-I:}

Investigadores: Roque Saltarén P, Tres asistentes del pregrado de $\mid M$.
Es un prototipo a pequeña escala. ver figura 2, cuya morfología geométrica se planteó semejante a la de un robot Puma, de seis grados de libertad y opciones de hombro izquierdo o derecho. El proyecto financiado por el CIFI, tuvo un costo de $\$ 400.000$.00 para la estructura mecánica.

Actualmente se van a adquirir las tarjetas electrónicas para el manejo de los servomotores DC., y se espera hacer funclonar el Robot para el segundo semestre del presente período.

Los desarrollos realizados para este prototipo no obstante su escala. implicaron la aplicación de los conceptos actuales del diseño de manipuladores, como es el caso del diseño mecánlco ligado a los problemas de la frecuencia natural de la estructura mecánica y su respuesta a las señales de control. En particular los efectos de flexibilidad y su efecto sobre la callidad del rendimlento del manipulador.

Simultáneamente durante el

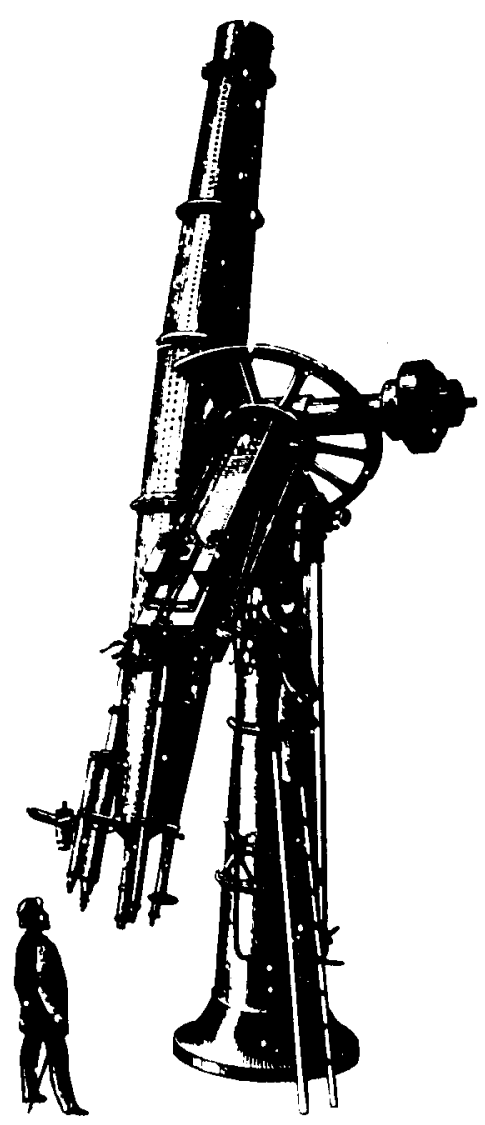


diseño y construcción del prototipo se estudiaron sus características cinemáticas y dlnámicas para lo cual fué necesario desarrollar programas computacionales de simulación como herramientas de trabajo.

\section{* Caso del Robot Manipulador ANDES-I.}

Investigador (DFAC.,Proyecto

PIDAI-Grupo RYP):

Roque Saltarén P. Tres asistentes de investigación pregrado-IM. por la disposición

Este proyecto de investigación (ver figura 3-4). Se inició en el año de 1989. Los primeros pasos comprendieron la exploración de la conveniencia de fabricar Robots en el país, y las necesidades del sector industrial de este tipo de equipos. A lo largo de la realización de visitas a varias Industrias tanto de los posibles fabricantes y proveedores de partes de Robots, como de usuarios, se tuvo una idea clara sobre la conveniencia de iniclar seriamente un diseño de un prototipo industrial que correspondiera a las expectativas de realización y demanda del secroe Industrial colombiano. En principlo se dlseñó un robot con capacidad de carga útil de $25 \mathrm{~kg}$. y seis grados de libertad, con accionamientos oleohidraúlicos.

Este diseño se tenía dlspuesto a finales de 1990 (Simulaciones cinematicas, dinámicas, de trayectorias, planos mecanicos para mecanizado etc.). La construcción del prototipo sufrió retrasos debido a las demoras presupuestales del proyecto PIDAl. No obstante se buscaron convenlos con posibles fabricantes del equipo, interesados igualmente en una transferencia de esta tecnologia por parte de la Universidad. Estas conversaciones con una empresa del sector metalmecánico no llegaron a objetivos concretos debido a las limitaciones económicas por parte de la Unlversldad y su contrapartida para la colaboración conjunta. Finalmente a princlpios de 1992 se tuvo luz verde de COLCIENCIAS, pero el interés captado en la Industrla se había entiblado, por esta razón se replanteó el prototipo de

\section{* Caso del Torno Multipropósito Convencional y su Conversión a Control Numérico}

Investigadores: Felipe Botero T, Roque Saltarén $P$, seis asistentes del pregrado de $\mathbb{M}$

No es difícil suponer que la modernizaclón del sector metalmecánlco del país y la adecuación de su capacidad de producción a las altas exlgenclas de calidad. cumplimiento $y$ competividad en un mercado local y externo conllevan a la inversión en sofisticados equipos de alta capacidad de producción y estrechas toleranclas de mecanizados. En contraste $\theta$ evidente que existen limitaclones para muchos empresarlos, en su capacidad de inversión en costosos blenes de capltal y la disposición de crédltos favorables, en consecuencla por qué no pensar en reallzar la conversión del numeroso parque de máquinas herramientas convenclonales, a máquinas herramlentas con facilldades reales de control numérico y comunicaclón con un ordenador ligado a las oflcinas de diseño de productos. Es plausible hoy en dia atacar este problema pues existen en el mercado numerosas alternativas como tarjetas de control multiejes, servomotores, sensores, software, el problema es integrar estos equipos coherentemente a un fin específlco como es el de automatizar una máquina herramienta.

Manipulador en especlal se optó por realizar un rediseño y cambiar hacia un equlpo de $15 \mathrm{~kg}$. de capacidad de carga útil $y$ acclonamientos eléctricos. Actualmente el $100 \%$ del problema de diseño se encuentra definldo $y$ se ha iniciado la adquisición de equipos y del Hardware necesarios, (Servo- motores DC. tarjetas de interfaces y control, etc.) y a finales de Jullo se Iniclará el mecanizado y emsamble de la estructura mecánica
En este marco de ldeas de Departamento de Ingeniería Mecánica así como el CIFI han iniclado trabajos de investigación, reallzándose Inversłones como son:

- La adqulsiclón de una máquina herramienta multipropósito de tres ejes $X, Y, Z$ (ver flgura -4 ).

- La adquisiclón de varladores de velocidad AC para el control del motor del husillo y motores de paso.

* Adquisiclón de transmisiones de potencia lineales. 
* La adiclón de espacio físico para estas investigaciones y la disposiclón de equipos computacionales para Investigaclón (PC 486).

De igual manera se realizó un estudio por parte del Director del Departamento de Ingeniería Mecánica, Rafael Beltrán P., sobre los aspectos docentes relacionados con los problemas actuales de la ingeniería de manufactura, la investlgación y desarrollo de nuevos productos y la formación de ingenieros con capacidades de actuar en un contexto altamente exigente en conocimientos de diseño mecánico y la integración del diseño a una producción automatizada de alta calidad, apoyándose en recursos razonablemente disponibles en nuestro medio como es el caso del software CAD/CAM. Este estudio dió lugar a la creación de una opción en Ingeniería de

Manufactura, con lo que se espera formalizar los aspectos docentes en estas áreas del conocimiento y realimentar la capacidad de los estudiantes a traves de investigaciones y los desarrollos que se irán concretando en proyectos que impliquen la automatización de sistemas mecánicos, la tecnología de mecanizado, control numérico y el diseño mecánico

\section{Conclusiones.}

Dentro de este panorama de activldades es importante notar una linea de acción claramente definida entre las actividades docente, investigativas y de realización de prototipos de software y fisicos de aplicación real a la industria, con un respaldo económico y de infraestructura conjunto de la facultad de Ingeniería, el CIFI, COLCIENCIAS y los Departamentos de Ingeniería Mecánica, de Sistemas y Eléctrica, cuyos resultados concretos estan claramente identificados. De otra parte es notable la vinculación de los estudiantes a las labores de Investigación y desarrollo, gracias a mecanismos como son por ejemplo los cursos de Proyectos Especiales, y el sentimiento evidente de su papel en realizaciones de trascendencia práctica y formativa.

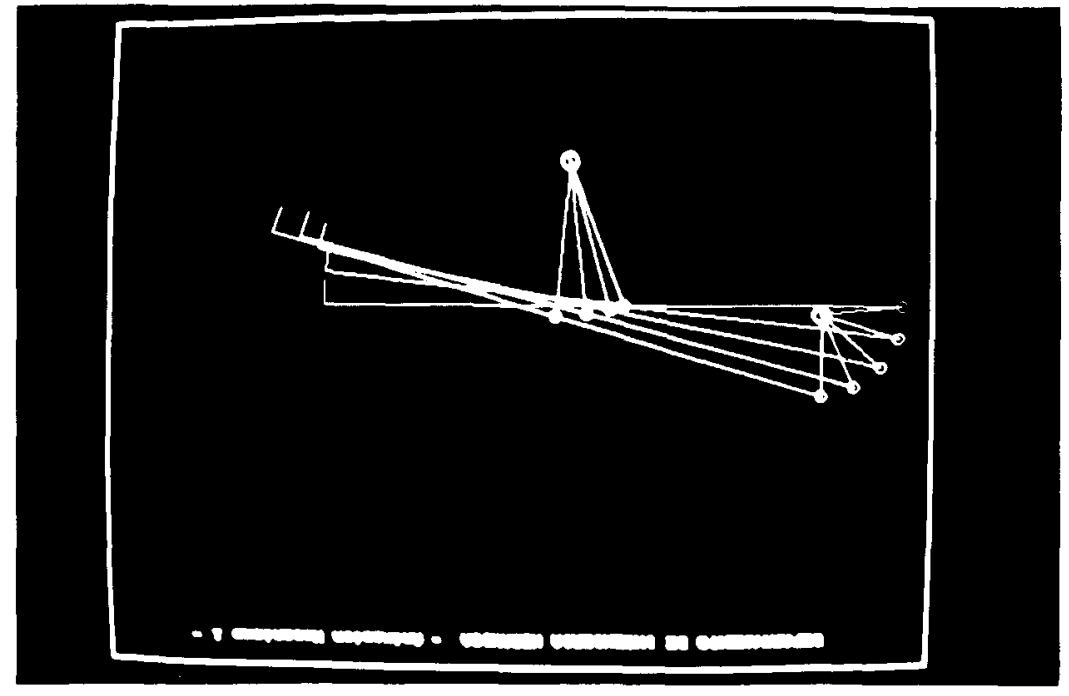

figura 1

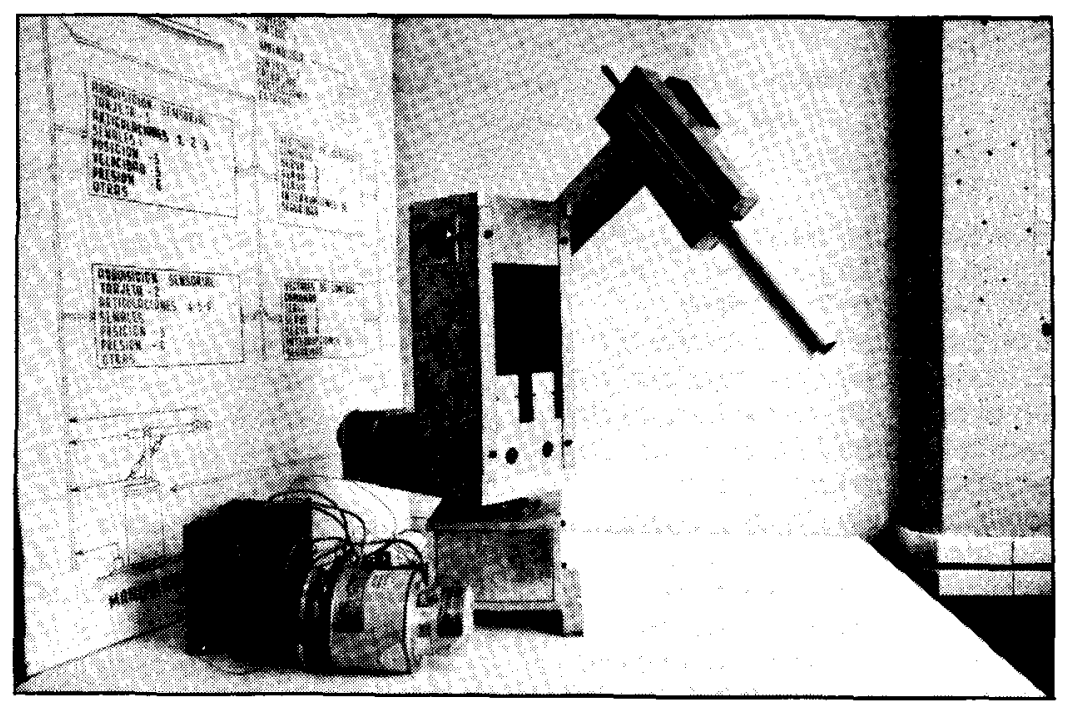

figura 2 


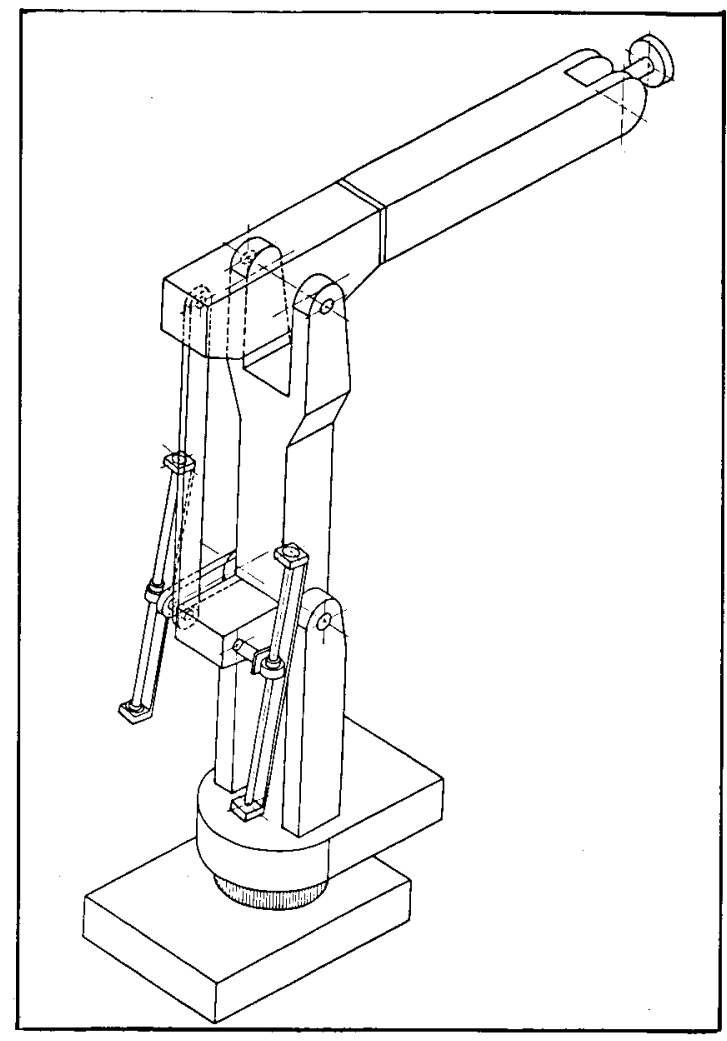

figura 3

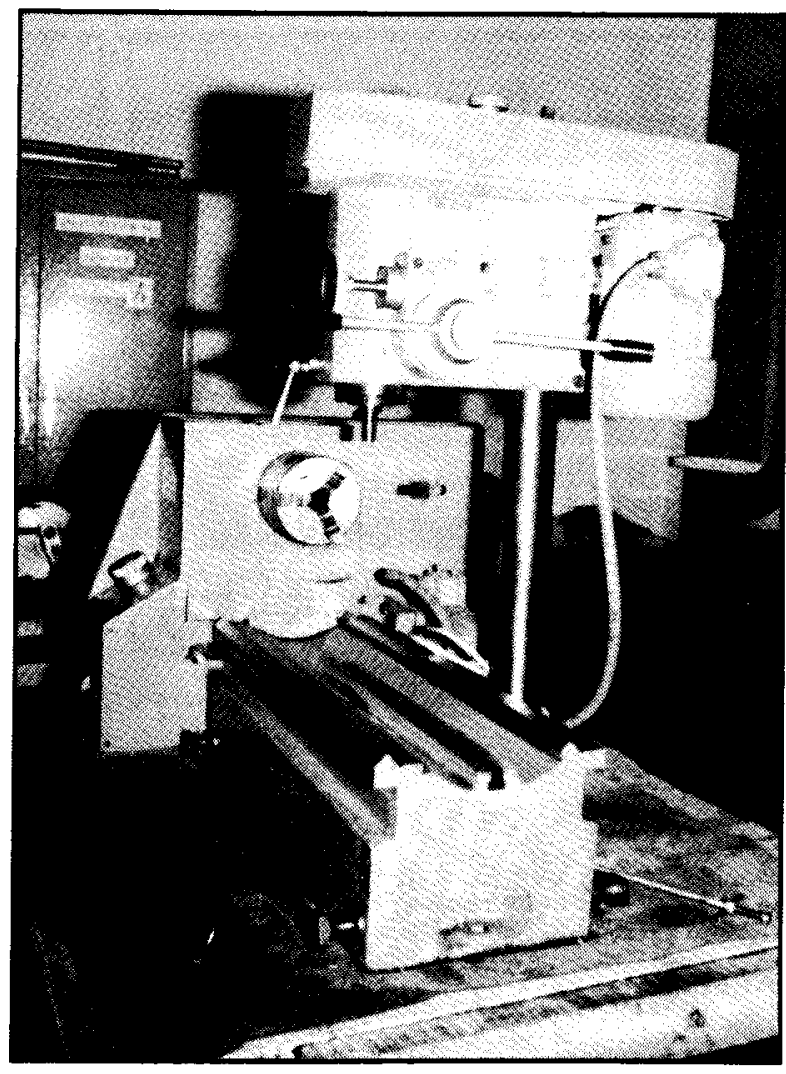

figura 4 

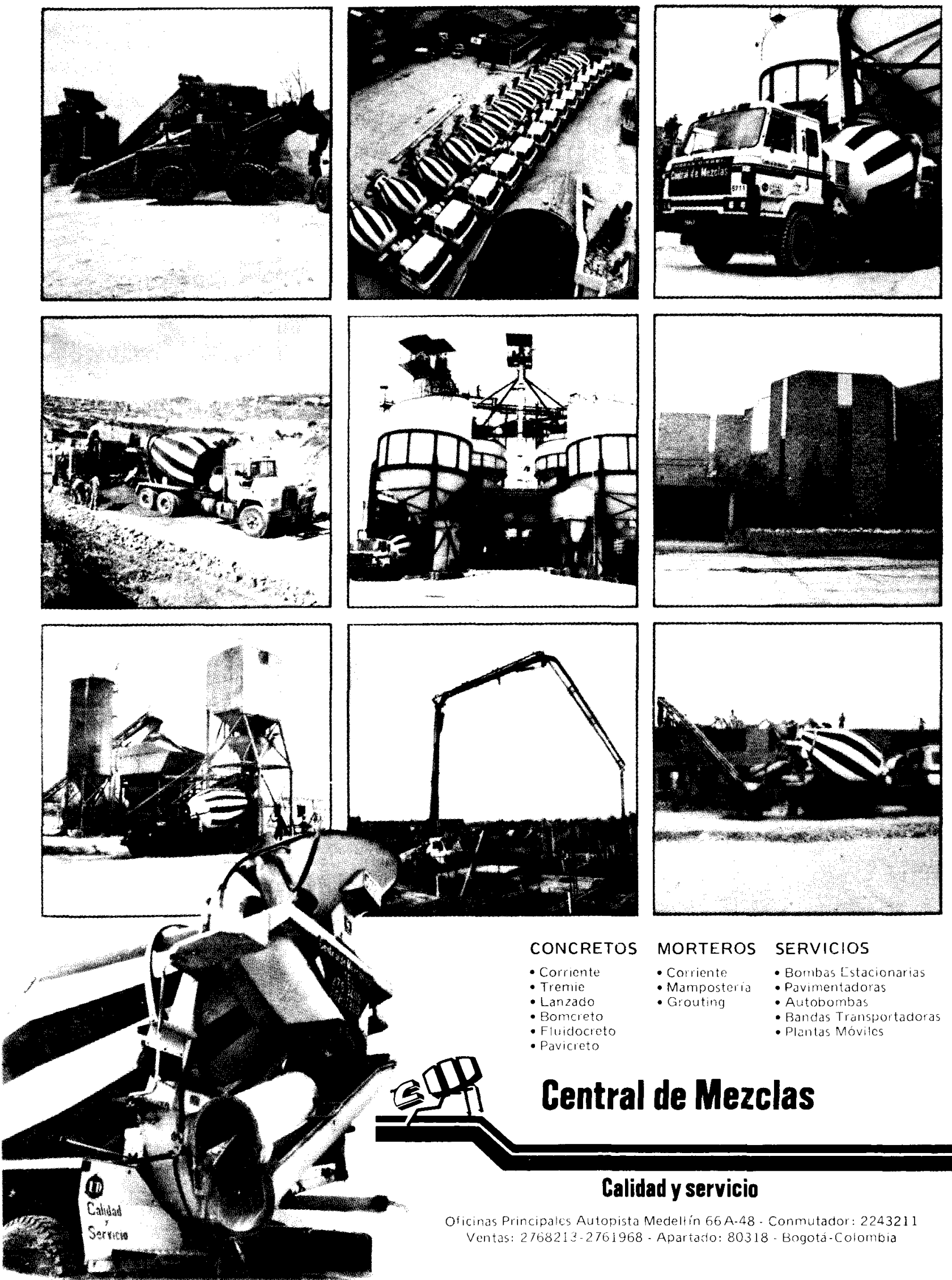

H

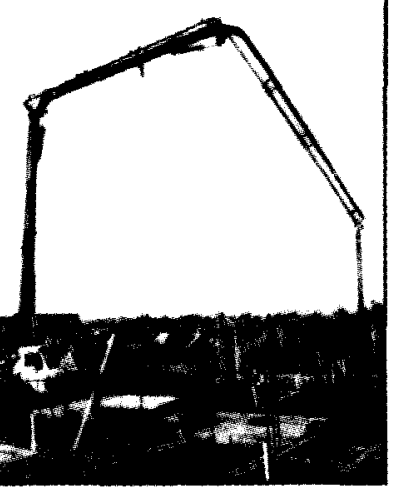

CONCRETOS

MORTEROS SERVICIOS

- Corriente

- Corriente

- Bombas Estacionarias

- Tremie - Mampostería - Pavimentadoras

- Lanzado

- Fiuidocreto

- Grouting

- Autonombas

- Bandas Transportadoras

- Pavicreto

\section{Central de Mezclas}

\section{Calidad y servicio}

Oficinas Principales Autopista Medellín 66 A-48 - Conmutador: 224321 Ventas: 2768213-2761968-Apartado: 80318-Bogotá-Colombia 


\section{T INGERSOLLRAND}
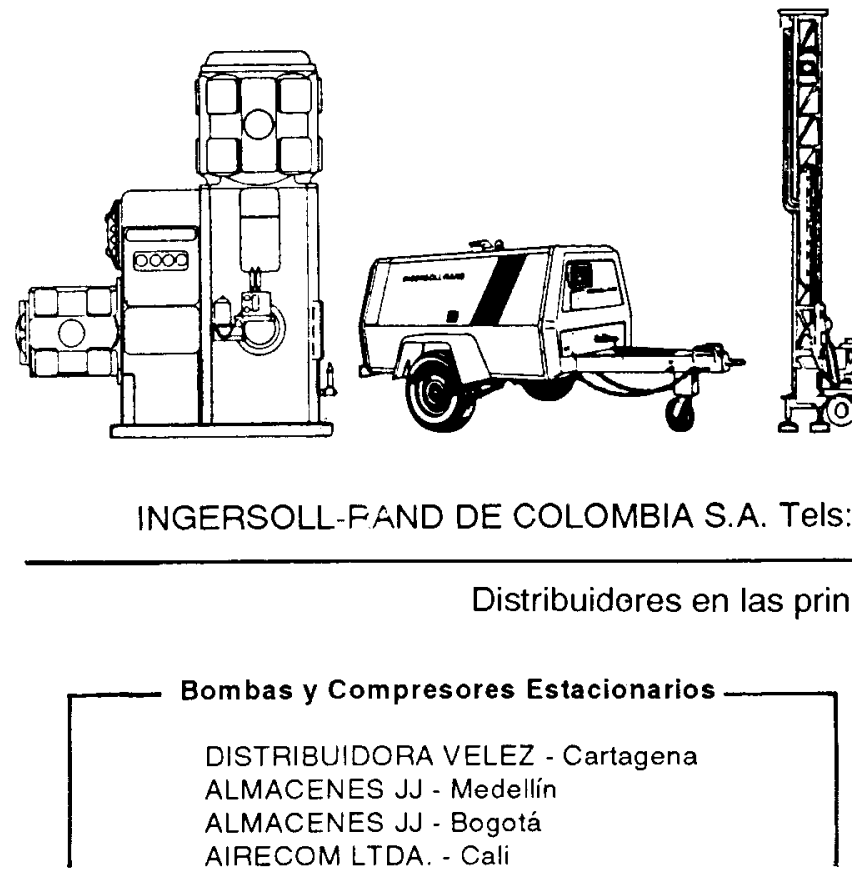

- Bombas centrífugas y reciprocantes

- Compresores de aire, estacionarios y portátiles

- Equipos para perforación con aire y lodo

- Maquinaria para construcción y mantenimiento de vías

- Plantas eléctricas portátiles de 20,30 y $50 \mathrm{Kw}$

INGERSOLL-FAND DE COLOMBIA S.A. Tels: 2191460 - 2191406 Fax: 6107589 BOGOTA

Distribuidores en las principales ciudades del país.

Bombas y Compresores Estacionarios

Equipos para Construcción

DISTRIBUIDORA VELEZ - Cartagena

ALMACENES JJ - Medellín

ALMACENES JJ - Bogotá

AIRECOM LTDA. - Cali

\section{plastitere}

\section{MAS DE 30 AÑOS DE PELICULA}

Película de polietileno de baja densidad.

- Película para cubrimiento de invernaderos.

- Película para empaque automático de leche, otros líquidos y granos.

- Bolsas para reempaque.

- Película transparente para usos industriales.

\section{¡Consúltenos!}

Plastilene S.A. 


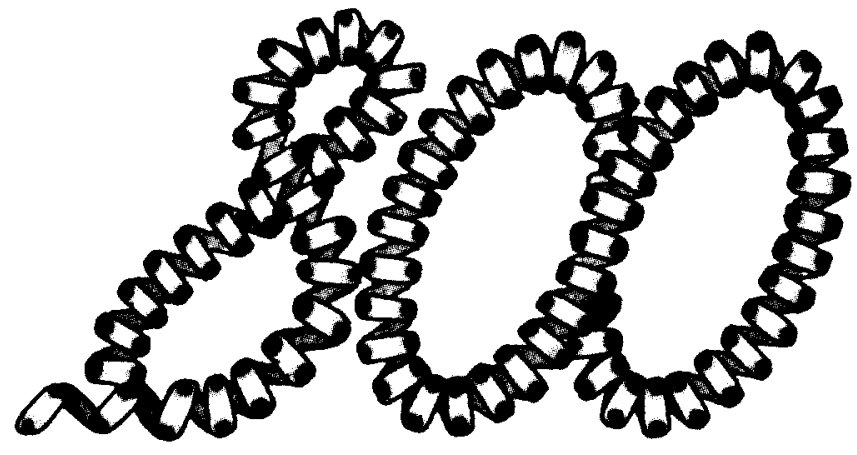

EnDinersClubestamos a tono con el progreso

9800-13611

Póngase a tono. Marque gratis desde cualquier lugar del país el $9800-13611$ y solicite la tarjeta de crédito Diners Club.

Si llama desde Santafé de Bogotá marque el 2813611.

Un servicio a tono con el progreso.

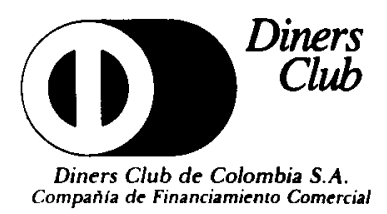

Solicite su tarjeta de crédito Diners Club
FERIVANIDO INIENIA \& EIA LITDAA Ingenieros Consultores

- Estudio de Suelos

- Perforaciones en Suelo y Roca

- Laboratorio de Suelos

- Ensayo de Densidad

\section{CONTEOON LIDA}

- Ensayo de Cilindros

- Diseños de Mezclas

- Pruebas de Carga

- Ensayos de Esclerómetro

y Ultrasonido

- Toma de Núcleos

Carrera 40 Ne 164 - 66 / 68

Tels: 6740673 - 6740723 - 6708084

6712947 - 6740730 - 6740732

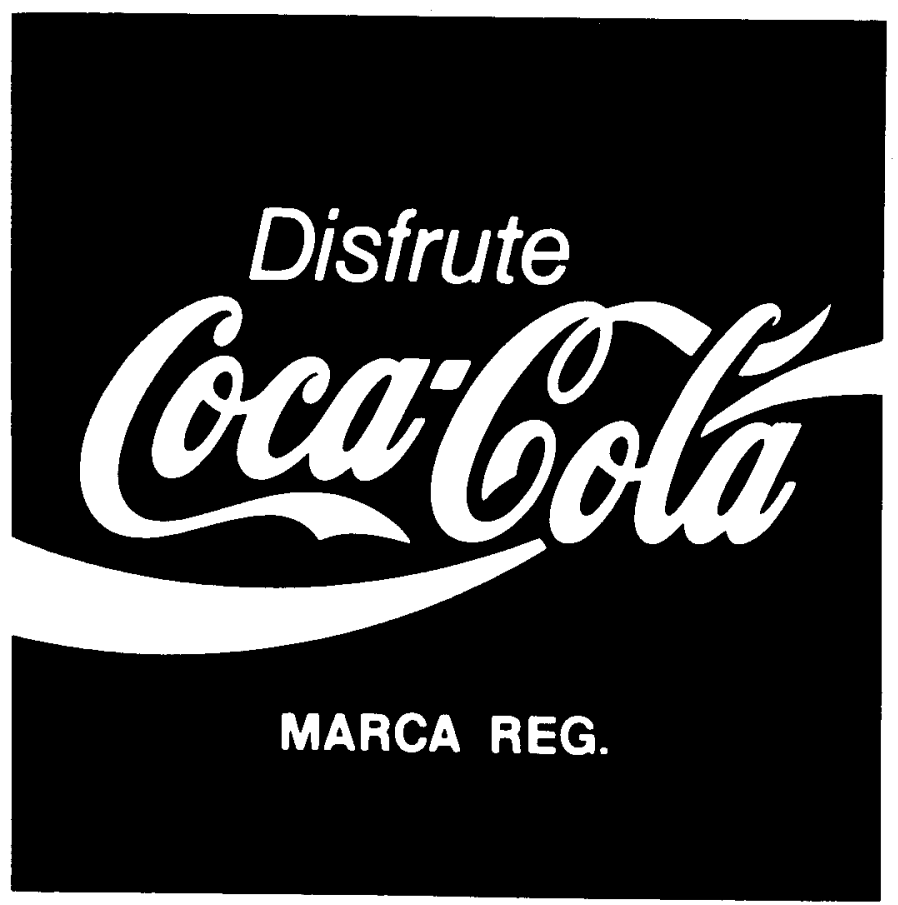




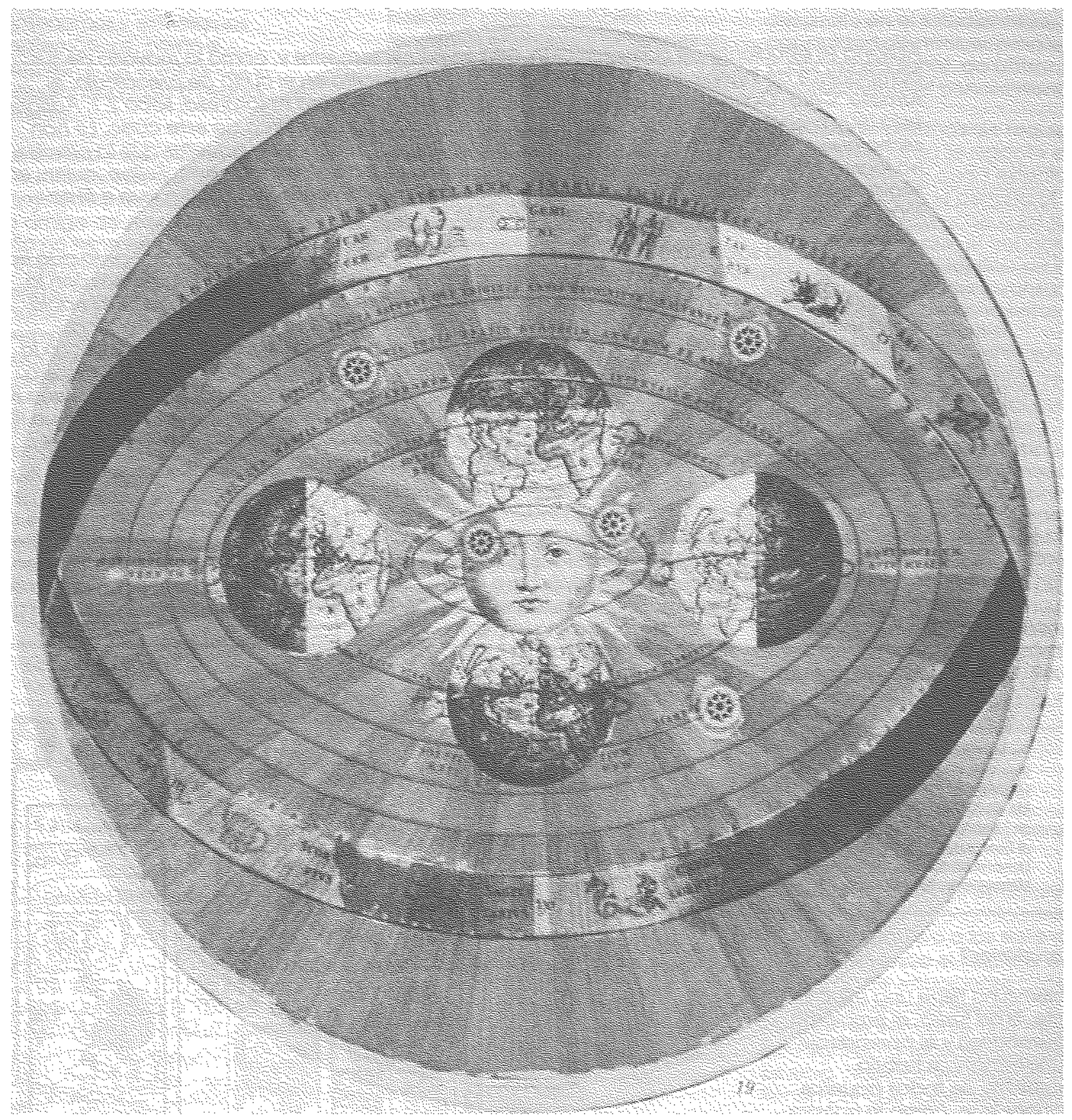

